Contrib. Plasma Phys. (2001),

\title{
Plasmon dispersion of a weakly degenerate nonideal one-component plasma
}

\author{
V. Golubnychiy ${ }^{1}$, M. Bonitz ${ }^{1}$, D. Kremp ${ }^{1}$, And M. Schlanges ${ }^{2}$ \\ ${ }^{1}$ Fachbereich Physik, Universität Rostock, Universitätsplatz 3, D-18051 Ros- \\ tock \\ ${ }^{2}$ Institut für Physik der Universität Greifswald, Domstrasse 10a, D-17489 Greif- \\ swald \\ e-mail:vova@mars.mpg.uni-rostock.de
}

\begin{abstract}
Classical Molecular Dynamics simulations (MD) for a one-component weakly degenerate plasma are presented. Using an effective quantum pair potential (Kelbg potential), the dynamic structure factor and the dispersion of Langmuir waves are computed. The influence of the coupling strength $\Gamma$ and degree of degeneracy $\rho \Lambda^{3}$ on these properties is discussed. The results are compared with predictions of mean-field theories.
\end{abstract}

The dielectric properties and the oscillation spectrum of charged particle systems have been studied in great detail for many decades, e.g. [1, 2, 3]. i). In the case of weakly correlated classical plasmas, where the coupling parameter $\Gamma=$ $(4 \pi \rho / 3)^{1 / 3} e^{2} / 4 \pi \epsilon_{0} k_{B} T$ ( $\rho$ is the electron density) is small, the appropriate description is given by the Vlasov theory. ii) On the other hand, weakly correlated quantum plasmas with $\rho \Lambda^{3}>1,(\Lambda$ is the DeBroglie wave length $)$, are well described by the random phase approximation (RPA). These two mean-field theories neglect correlation effects, in particular they do not take into account plasmon damping due to collisions. The latter effect becomes essential for increasing coupling, $\Gamma>1$. iii) Strong coupling in classical plasmas can be efficiently studied using molecular dynamics simulations, e.g. [2, 3]. However, this powerful method is not applicable to quantum systems. iv) Alternative approaches to the dielectric properties of quantum systems, such as density functional or quantum kinetic theory, e.g. [1, [1, are usually successful up to moderate coupling, $r_{s} \equiv \bar{r} / a_{B}<1$, where $\bar{r}$ and $a_{B}$ denote the mean inter-particle distance and the Bohr radius, respectively.

This brief survey shows that plasmas in the region of moderate coupling and degeneracy are difficult to describe reliably by means of established methods. In this paper, we analyze the dynamic structure factor and the Langmuir plasmon dispersion for weakly degenerate plasmas, $\rho \Lambda^{3} \leq 1$, at intermediate coupling, $\Gamma \leq 4$. For these parameters, it is possible to perform classical MD simulations using effective quantum pair potentials. Such potentials have been rigorously derived from the two-particle Slater sum using Morita's method by Kelbg and co-workers [5]. In this paper we alternatively use the Kelbg potential and the Coulomb potential which allows us to study the influence of quantum diffraction effects on the dynamic properties of an OCP. We find that quantum effects tend to soften the wave vector dispersion.

The dielectric and dynamic properties of an N-particle system can be derived from the density-density correlation function $A(\vec{k}, t)$, which is defined as [1]:

$$
A(\vec{k}, t)=\frac{1}{N}\left\langle\rho_{\vec{k}}(t) \rho_{-\vec{k}}(0)\right\rangle, \quad \rho_{\vec{k}}(t)=\sum_{i=1}^{N} e^{i \vec{k} \vec{r}_{i}(t)}
$$


Here, $\rho_{\vec{k}}(t)$ is the spatial Fourier component of the density which is computed from the trajectories $\vec{r}_{i}(t)$ of all particles. The dynamical structure factor is just the Fourier transformation of the density-density correlation function

$$
S(\vec{k}, \omega)=\frac{1}{2 \pi} \int_{-\infty}^{+\infty} d t e^{i \omega t} A(\vec{k}, t)
$$

The trajectories of particles $\vec{r}_{i}(t)$ can be directly obtained by numerical solving classical (Newton's) equations of motion for the interacting N-body system which include all binary Coulomb forces, e.g. 2]. To include quantum diffraction effects, one can replace the Coulomb potential by an effective quantum potential which was derived by Kelbg [5]

$$
U_{\mathrm{KELBG}}(r, T)=4 \pi e^{2}\left(\frac{1-\exp \left(-r^{2} / \lambda^{2}\right)}{r}+\frac{\sqrt{\pi}}{\lambda} \operatorname{erfc}(r / \lambda)\right), \quad \lambda=\frac{\Lambda}{\sqrt{2 \pi}} .
$$

The Kelbg potential (3) is finite at zero distance, correctly accounting for the Heisenberg uncertainty. Most importantly, it is the exact quantum potential in the case of small $\Gamma$ and we expect that, also at moderate coupling, it accounts for the dominant quantum effects. This potential is used in the MD simulations shown below. Furthermore, the long range part of the interaction was computed in standard way by using Ewald summation procedure and 3-dimensional lookup tables, for details cf. [6].

We have performed a series of simulations for varying values of $\Gamma$ and $\rho \Lambda^{3}$, using the Coulomb and the Kelbg potential, respectively. Fig. 1. shows the dynamical structure factors $S(q, \omega)$, where $q$ is the dimensionless wave number, $q=|\mathbf{k}| \bar{r}$. In the figure, also the results for Vlasov and

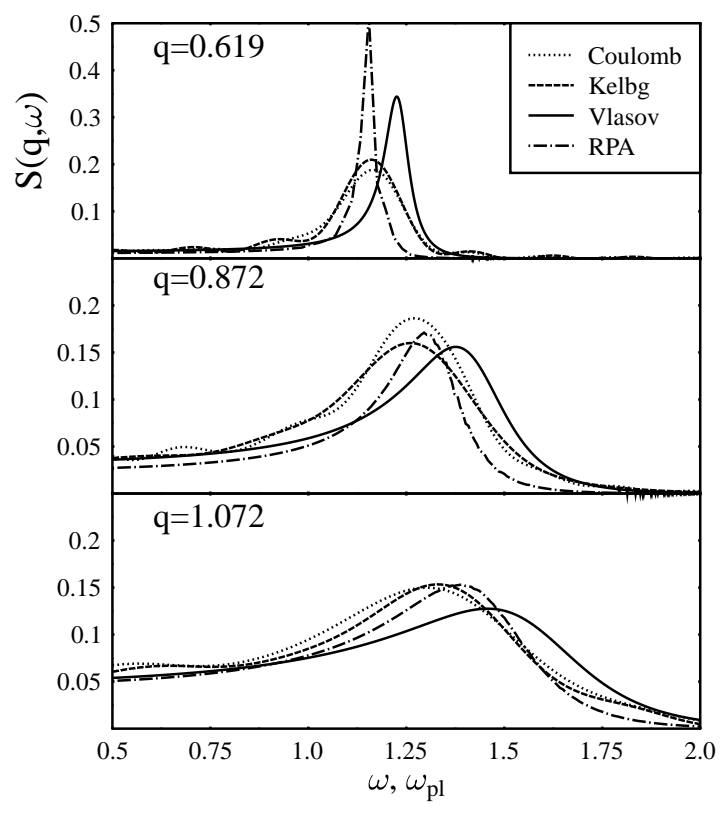

Fig. 1: Dynamical structure factor an OCP at $\Gamma=1$ and $\rho \Lambda^{3}=0.1$ from MD simulation with Coulomb and Kelbg potential.

RPA theories are plotted. The structure factor is clearly peaked at the frequency of the optical (Langmuir) plasmon. The width of the peak, i.e. the damping of the oscillations, increases rapidly with growing wave number. As expected, the mean-field results have narrower plasmon peaks which is due to the neglect of inter-particle collisions - their widths is solely due to collisionless Landau damping. In contrast, the MD results fully include Landau and collisional damping. Further, as a result of the increased damping, the plasmon peak obtained in the MD simulations is shifted to lower 
frequencies. Comparison of the simulation results with Coulomb and Kelbg potential shows only slight differences which are most pronounced at large wave numbers. The reason is that the interaction potentials differ only at small distances.

Increasing the degeneracy, we observe that the peak of the structure factor moves towards lower frequencies. A more quantitative comparison can be made by following the plasmon peak when the wave number is varied. The resulting wave number dispersion of Langmuir oscillations is shown in Fig. 2 for a dense OCP with three values of the coupling strength. In the limit of small wave numbers, all models tend to the same frequency, the plasma frequency $\omega_{p l}$. (Notice that simulation results are available only for finite wavenumbers, $q \geq q_{\min }$, where $q_{\min }$ is limited by the size of the simulation box.) With increasing wave number, the frequency is expected to grow. In particular, the Vlasov theory predicts, for small wave numbers, $k r_{D}<1$, the behavior $\omega_{0}(q)=\omega_{p l} \sqrt{1+q^{2} / \Gamma}$. Our results show that, for $\Gamma=$ 0.5 and $q \leq 0.7$ this formula works satisfactorily. However, for larger wave num-
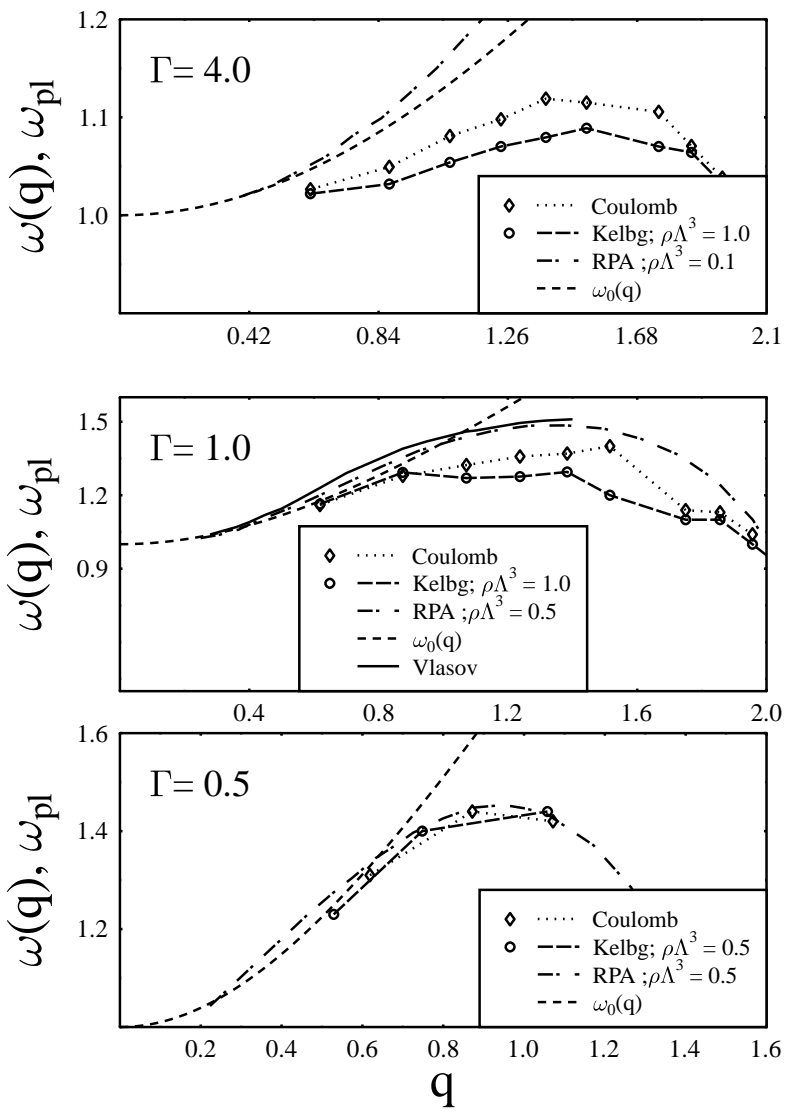

Fig. 2: Wave number dispersion of Langmuir waves for various coupling and degeneracy parameter from MD simulations with the Coulomb and Kelbg potentials. Furthermore, results from the Vlasov and RPA approximations are shown. $\omega_{0}(q)$ denotes the well-known analytical low $q$ limit of the Vlasov theory, see text.

bers, the plasmon frequency grows more slowly until it reaches a maximum around $q \approx 1$. For $\Gamma=0.5$, the plasmon dispersion is overall well reproduced by the RPA result which we found to be significantly more accurate than the Vlasov theory.

Let us now consider larger values of the coupling parameter $\Gamma$, cf. the upper two figure parts. Here, the differences between the mean field theories and the simulations are becoming more prononounced: the MD results yield an essentially lower plasmon frequency than the RPA and the Vlasov theory. Further, with increasing coupling strength the dispersion curves become more flat and their maxima decrease. Finally, let us analyze the influence of quantum effects by comparing the simulations with the Coulomb and Kelbg potentials: we generally find that the dispersion curves with the Kelbg potential are below those for the Coulomb case. This difference grows 
with increasing plasma degeneracy at constant $\Gamma$ confirming that this is a quantum diffraction effect.

Finally, in Fig. 3 we study the dependence of the dispersion relations for the Kelbg potential on the degeneracy parameter $\rho \Lambda^{3}$ for two values of the coupling parameter. Again we confirm that increasing degeneracy leads to a lowering (softening) of the plasmon dispersion. One may attempt to construct a fit for the plasmon dispersion which improves the above approximation $\omega_{0}(q)$. Fig. 3 shows the best fit constructed according to the ansatz $\omega(q) / \omega_{p l}=\left(1+a q^{2}+\right.$ $\left.b q^{4}\right)^{1 / 2}$, where the points with smallest wave vector were given the largest weight [7]. The fit parameters for two values of $\Gamma$ and various degeneracies are summarized in the table.

In summary, we have presented classical MD simulations with the Kelbg potential which allowed us to compute the optical plasmon dispersion of a weakly degenerate moderately correlated one-component plasma. The main conclusion is that increase of coupling and degeneracy influ-

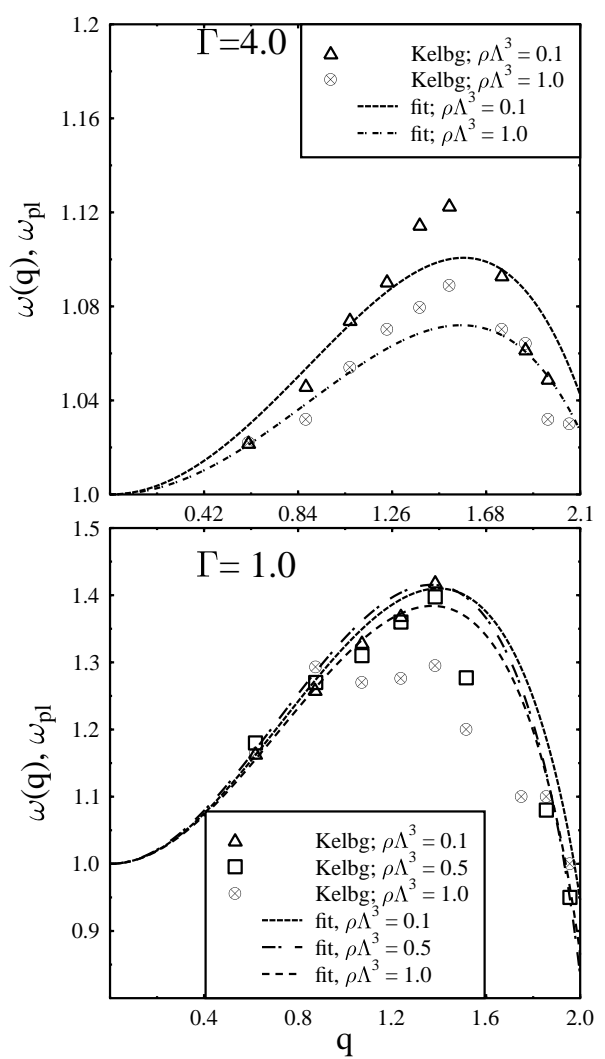

Fig. 3: Optical dispersion relations for different coupling and degeneracy parameter for the OCP with Kelbg potential. Symbols are MD results, lines the best fits.

ence the dispersion in similar way: they reduce the plasmon frequency. The physical reason is simple: with increasing $\Gamma$, the Coulomb interaction is screened more and more. Thus, the binary interaction is dominated by short-range effects which tends to soften the collective mode. Similarly, increase of the degeneracy leads to a growth of the electron wave length which, again, increases the efficiency of short-range binary interaction. 
Tab. 1: Fit parameters of the Langmuir dispersion curves shown on Fig. 3. The fit equation was taken in the form $\omega(q) / \omega_{p l}=\left(1+a q^{2}+b q^{4}\right)^{1 / 2} . q$ is in units of $\bar{r}$. The fit parameters for $\Gamma=1$ and $\rho \Lambda^{3}=0.1$ are less reliable, because of the absence of data for big wave vectors.

\begin{tabular}{|c|c|c|c|}
\hline$\Gamma$ & $\rho \Lambda^{3}$ & $\mathrm{a}$ & $\mathrm{b}$ \\
\hline 1.0 & 0.1 & $1.013 \pm 0.031$ & $-0.260 \pm 0.023$ \\
1.0 & 0.5 & $1.074 \pm 0.041$ & $-0.288 \pm 0.013$ \\
1.0 & 1.0 & $0.975 \pm 0.055$ & $-0.259 \pm 0.018$ \\
\hline 4.0 & 0.1 & $0.169 \pm 0.015$ & $-0.034 \pm 0.006$ \\
4.0 & 1.0 & $0.121 \pm 0.007$ & $-0.025 \pm 0.003$ \\
\hline
\end{tabular}

\section{Acknowledgements}

This work is supported by the DFG (Schwerpunkt "Laserfelder") and a grant for CPU time at the NIC Jülich.

\section{References}

[1] For an overview, see e.g. S. Ichimaru, "Statistical Plasma Physics" Vol.I, AddisonWesley Publishing Company, 1992

[2] J.M. Caillol, D. Levesque, J.J. Weis, and J.P. Hansen, J. Stat. Phys 28, 325 (1982)

[3] W. Ebeling, G.E. Norman, A.A. Valuev, and I.A. Valuev, Contr. Plasma Phys. 39, 61 (1999)

[4] N. Kwong, and M. Bonitz, Phys. Rev. Lett. 84, 1768 (2000)

[5] G. Kelbg, Ann. Physik, 12, 219 (1963); 13, 354; 14, 394 (1964).

[6] V. Golubnichiy, M. Bonitz, D. Kremp, and M. Schlanges, subm. to Phys. Rev. E

[7] Obviously, the wave vector dispersion of the simulation data is more complex than this formula. But to further refine the ansatz, more simulation data would be required. To improve the present fit, the MD-data for various $q$ were weighted by the values of the plasmon damping for that $q$-value. 